\title{
Early effects of androgen deprivation on bone and mineral homeostasis in adult men: a prospective cohort study
}

\author{
Rougin Khalil', Leen Antonio ${ }^{10}$, Michaël R Laurent ${ }^{2}$, Karel David', Na Ri Kim¹, Pieter Evenepoel ${ }^{3}$, \\ Anton Eisenhauer ${ }^{4}$, Alexander Heuser ${ }^{4}$, Etienne Cavalier $^{5}$, Sundeep Khosla ${ }^{6}$, Frank Claessens ${ }^{7}$, \\ Dirk Vanderschueren ${ }^{1}$ and Brigitte Decallonne ${ }^{1}$
}

${ }^{1}$ Clinical and Experimental Endocrinology, Department of Chronic Diseases, Metabolism and Aging, KU Leuven, Leuven, Belgium, ${ }^{2}$ Center for Metabolic Bone Diseases, Geriatrics Department, University Hospitals Leuven, Leuven, Belgium,

${ }^{3}$ Nephrology and Renal Transplantation Research Group, Department of Microbiology, Immunology and Transplantation, KU Leuven, Leuven, Belgium, ${ }^{4}$ GEOMAR Helmholtz Centre for Ocean Research Kiel, Kiel, Germany, ${ }^{5}$ Department of Clinical Chemistry, University of Liège (ULg CHU), Liège, Belgium, ${ }^{6}$ Kogod Center on Aging and Division of Endocrinology and Metabolism, Mayo Clinic College of Medicine and Science, Rochester, Minnesota, USA, and ${ }^{7}$ Molecular Endocrinology Laboratory, Department of Cellular and Molecular Medicine, KU Leuven, Leuven, Belgium

Correspondence should be addressed to B Decallonne Email brigitte.decallonne@ uzleuven.be

\begin{abstract}
Objective: Long-term androgen deprivation therapy (ADT) negatively influences bone. The short-term effects on bone and mineral homeostasis are less known. Therefore, we aimed to investigate the early effects of ADT on calcium/ phosphate homeostasis and bone turnover.

Design: Prospective cohort study.

Methods: Eugonadal adult, male sex offenders, who were referred for ADT to the endocrine outpatient clinic, received cyproterone acetate. Changes in blood markers of calcium/phosphate homeostasis and bone turnover between baseline and first follow-up visit were studied.

Results: Of 26 screened patients, 17 were included. The median age was 44 (range 20-75) years. The median time interval between baseline and first follow-up was 13 (6-27) weeks. Compared to baseline, an $81 \%$ decrease was observed for median total testosterone (to $3.4 \mathrm{nmol} / \mathrm{L}(0.4-12.2) ; P<0.0001$ ) and free testosterone (to $0.06 \mathrm{nmol} / \mathrm{L}$ (0.01-0.18); $P<0.0001$ ). Median total estradiol decreased by $71 \%$ (to $17.6 \mathrm{pmol} / \mathrm{L}(4.7-35.6) ; P<0.0001)$. Increased serum calcium $(P<0.0001)$ and phosphate $(P=0.0016)$ was observed, paralleled by decreased PTH $(P=0.0156)$ and 1,25-dihydroxyvitamin $\mathrm{D}_{3}(P=0.0134)$. The stable calcium isotope ratio $\left(8^{44 / 42} \mathrm{Ca}\right)$ decreased $(P=0.0458)$, indicating net calcium loss from bone. Bone-specific alkaline phosphatase and osteocalcin decreased $(P<0.0001$ and $P=0.0056$, respectively), periostin tended to decrease $(P=0.0500)$, whereas sclerostin increased $(P<0.0001)$, indicating suppressed bone formation. Serum bone resorption markers (TRAP, CTX) were unaltered.

Conclusions: In adult men, calcium release from the skeleton occurs early following sex steroid deprivation, reflecting early bone resorption. The increase of sclerostin and reduction of bone formation markers, without changes in resorption markers, suggests a dominant negative effect on bone formation in the acute phase.
\end{abstract}




\section{Introduction}

In men, testosterone (TS) and estradiol (E2) are not only important regulators of bone mass acquisition during puberty, but they are also essential for the maintenance of adult bone (1). It is well known that male hypogonadism is associated with bone loss. Accordingly, in prostate cancer patients androgen deprivation therapy (ADT) induces bone loss, thereby increasing the risk for osteoporosis and fractures $(2,3,4,5,6,7,8,9)$. However, in case of metastatic prostate cancer the impact of ADT per se on bone homeostasis could be obscured. ADT is also used in male sex offenders with paraphilic or hypersexual disorder (10), either by using androgen receptor (AR) antagonists or GnRH agonists or antagonists. In contrast to prostate cancer patients, this patient group is generally younger, has less comorbidities, and is expected to need longterm treatment with ADT $(11,12)$. However, research on adverse health effects of ADT is limited in these patients and only a few studies included data on bone health $(3,4$, $5,6,8)$. Moreover, while the long-term effects of androgen deprivation on bone have been extensively investigated, mainly in prostate cancer patients, studies on the shortterm impact of changes in sex steroids on bone and mineral homeostasis are limited.

In patients treated with ADT, serum bone turnover markers (BTMs) can be used to detect changes in bone homeostasis, thereby enabling the assessment of both bone formation and resorption. Classical formation markers include $\mathrm{N}$-terminal propeptide of type 1 collagen (P1NP), which is released during formation of type I collagen; bone alkaline phosphatase (BAP), a protein found on the surface of osteoblasts reflecting their activity; and osteocalcin, produced by osteoblasts and incorporated into the bone matrix. Bone resorption markers include C-or N-terminal telopeptide of type I collagen (CTX or NTX), degradation products of type I collagen; and tartrate-resistant acid phosphatase 5b (TRAP), an enzyme produced by osteoclasts that reflects their number (13, 14). These BTMs are blood based and relatively easy to measure, and can therefore be repeatedly assessed in the same patient (13). However, BTMs have disadvantages as well. They can be affected by sex, age, body weight, circadian rhythm, food intake, exercise, renal or liver function, recent bone fracture and lifestyle (13).

Several studies have shown that long-term sex steroid deprivation with GnRH agonists in adult men increases both bone resorption and formation markers, by stimulating bone remodeling, with a dominant effect on resorption, leading to a negative bone mineral balance $(15,16)$. In the acute phase of ADT, however, the group of Khosla revealed decreased osteocalcin and P1NP levels 3 weeks following GnRH agonist treatment combined with an aromatase inhibitor, suggesting suppressed bone formation. Furthermore, supplementation with either TS and/or E2 showed that E2 is mainly involved in the suppression of bone resorption, while TS mainly stimulates bone formation (17).

In this study, we aimed to better understand the early effects of ADT on bone and mineral homeostasis. Therefore, we studied the serum calcium/phosphate response, and the change in calciotropic hormones and bone turnover markers in a prospective cohort of adult male sex offenders referred for initiation of ADT.

We hypothesized that in the early phase of androgen deprivation bone resorption is increased, whereas bone formation is reduced, causing a negative bone balance, which subsequently influences mineral metabolism.

\section{Subjects and methods}

\section{Participants and study design}

Between July 2012 and June 2016, male sex offenders aged $\geq 18$ years were asked to participate in an observational study at the University Hospitals Leuven, Belgium, Department of Endocrinology. All men were referred by their treating psychiatrist for medical evaluation before and during ADT as adjuvant therapy to psychotherapy. ADT consisted of daily oral intake of cyproterone acetate (CPA, $50 \mathrm{mg} /$ day), in line with the guidelines for management of male sex offenders $(18,19)$. CPA is an AR antagonist that also functions as a progestagen, thus also decreasing LH and FSH and suppressing endogenous sex steroid production.

The following clinical and biochemical data were extracted from the medical files: height, weight, smoking status, history of diabetes mellitus, albumin, hemoglobin, renal function (eGFR, cystatin C), liver function (alkaline phosphatase (ALP); aspartate aminotransferase (AST); alanine transaminase (ALT); gamma-glutamyl transferase (GGT)), and maintenance therapy. Patients under bone anti-resorptive treatment or medication interfering with sex steroid metabolism or calcium/phosphate homeostasis, except for calcium and/or vitamin D supplements, were excluded. Men with hypogonadism prior to the start of ADT (defined by a total $\mathrm{T} \leq 7 \mathrm{nmol} / \mathrm{L}$, measured between 8:00 $\mathrm{h}$ and 10:00 $\mathrm{h}$ ) were also excluded, as well as men without decrease in TS under CPA treatment, suggestive for non-compliance. As shown in Fig. 1, 26 participants 
$\mathrm{n}=26$ subjects screened prior to ADT

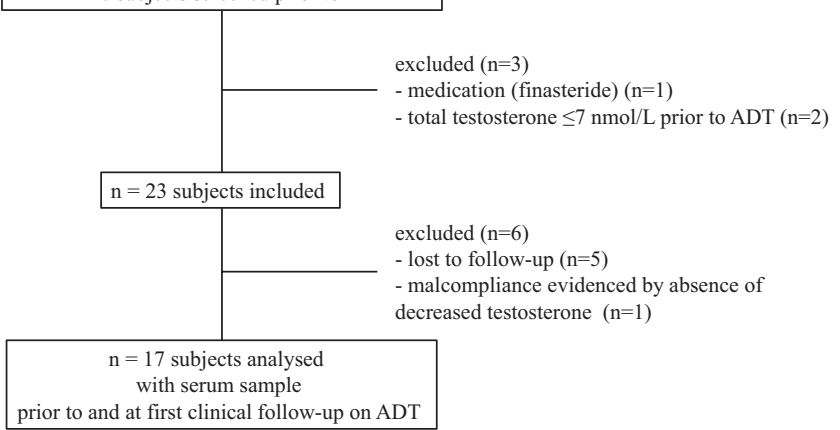

\section{Figure 1}

Study flow diagram.

were screened and 17 participants were eligible for final analysis. The Ethical Committee of the University Hospitals, Leuven, approved the study (ClinicalTrials. gov number NCT02434562). All participants gave written informed consent.

\section{Blood sampling and laboratory measurements}

A morning blood sample was taken prior to treatment initiation and at first clinical follow-up 2-4 months post initiation as part of standard clinical care. All measurements were performed in single-assay runs.

\section{Gonadal axis}

Total TS and total E2 were measured by liquid chromatography-tandem mass spectrometry as previously described $(20,21)$. SHBG was measured using the modular E170 platform electrochemiluminescence immunoassay (Roche Diagnostics). Free TS was calculated using the Vermeulen formula (22). LH and FSH were assessed by an electrochemiluminescence immunoassay (HITACHI/ Roche - COBAS 8000).

\section{Calcium and phosphate homeostasis}

Calcium, phosphate, and albumin were measured on a HITACHI/Roche - COBAS c702. Calcium was corrected for albumin using the following formula: serum calcium $+0.8 \times(4-$ serum albumin). 25-hydroxyvitamin D, 1,25-dihydroxyvitamin $\mathrm{D}_{3}$ and 24,25-dihydroxyvitamin $\mathrm{D}_{3}$ were measured by liquid chromatography-tandem mass spectrometry $(23,24)$. Vitamin D-binding protein (DBP) was measured by single radial immunodiffusion, as previously described (25). Free vitamin D was calculated using the Vermeulen formula (22). Third generation PTH and intact fibroblast growth factor 23 (FGF23) were measured with the Liaison automate (Diasorin, Saluggia, Italy).

\section{Bone turnover markers}

BAP, osteocalcin, P1NP, and TRAP were measured with the IDS iSYS platform (Boldon, UK). Measurement of sclerostin (TECO medical, Sissach, Switzerland) and periostin (Biomedica, Vienna, Austria) was performed by ELISA. CTX was assessed by an electrochemiluminescence immunoassay (HITACHI/Roche - COBAS 8000).

\section{Stable calcium isotopes}

Stable calcium isotope measurements were performed on a MC-ICP-MS (Neptune plus, Thermo Fisher Scientific) at the mass spectrometer facilities of the GEOMAR Helmholtz Centre for Ocean Research Kiel in Germany, as previously described (26). NIST SRM 915a was used as a reference material. The isotopic composition is reported as $\delta^{44 / 42} \mathrm{Ca}$ in parts per thousand (\%o). Calcium consists of six stable natural isotopes, with a mass from 40 to 48 atomic mass units. During bone formation, the lighter isotopes are preferentially incorporated into bone, while there is no isotope preference during bone resorption. Thus, in case of a positive bone mineral balance, a serum shift toward heavier values will occur, while lighter isotopes become more abundant in case of a negative bone mineral balance (27). Due to sample availability, the stable calcium isotope ratio was determined on 12 out of 17 patients.

\section{Statistical analysis}

Values are expressed as median (range). To analyze changes in biochemical parameters after initiation of ADT, normality was first assessed with the D'Agostino \& Pearson normality test. If data were normally distributed, a paired $t$-test was applied, otherwise the Wilcoxon matched-pairs signed-rank test was used to determine statistical significance (two-tailed $P<0.05$ ). All analyses were performed using GraphPad Prism (version 6.07).

\section{Results}

\section{Effect of androgen deprivation on general parameters and on the gonadal axis}

At baseline, the median age of the cohort was 44 years (20-75), with a median BMI of $24.6 \mathrm{~kg} / \mathrm{m}^{2}$ 
(17.9-31.7). Eleven patients (65\%) were active smokers or had a history of smoking. None of the patients had diabetes mellitus. The average time interval between the start of therapy and the first follow-up visit was 13 weeks (6-27). No changes were observed in BMI, kidney function, or liver function. A decrease in albumin and hemoglobin, a proxy for androgen deficiency, as well as a decrease of total ALP was observed (Table 1).

At the first follow-up visit, androgen deprivation caused an $81 \%$ decrease in total TS as well as free TS on average. E2 concentrations decreased 71\% and SHBG decreased 30\% on average. Gonadotropins were lowered as well (Fig. 2 and Table 1).

\section{Effect of androgen deprivation on serum calcium and phosphate homeostasis}

Albumin-corrected serum calcium and phosphate concentrations increased after ADT (Fig. 3 and Table 2). PTH and 1,25-dihydroxyvitamin $\mathrm{D}_{3}$ decreased, while 25-hydroxyvitamin $\mathrm{D}_{3}$ increased. However, serum DBP also increased, leading to similar free 25-hydroxyvitamin $\mathrm{D}_{3}$ under ADT, while free 1,25-dihydroxyvitamin $\mathrm{D}_{3}$ decreased to a greater extent. 24,25-dihyroxyvitamin $\mathrm{D}_{3}$ and 25D:24,25D ratio did not change significantly. No change was observed for serum FGF23 (Table 2).

\section{Effect of androgen deprivation on bone homeostasis}

The serum levels of the bone resorption markers TRAP and CTX were not affected by ADT. The serum levels of the bone formation markers BAP and osteocalcin were decreased (Fig. 4 and Table 2), while P1NP was not altered. Serum sclerostin clearly increased, while periostin levels tended to decrease following ADT. Finally, a decrease in the calcium isotope ratio was observed, indicating a negative bone mineral balance (Fig. 4 and Table 2).

\section{Discussion}

We observed that ADT with CPA in adult males is associated with a negative bone mineral balance, releasing calcium and phosphate from the skeleton already after approximately 3 months of treatment, with a compensatory decline in serum PTH and 1,25-dihydroxyvitamin $\mathrm{D}_{3}$. Accordingly, the stable calcium isotope ratio, allowing the sensitive detection of early calcium loss from bone, decreased even while the bone resorption markers CTX and TRAP were unaltered. This mild bone resorption was paralleled by a clear suppression of bone formation in the early phase of androgen deprivation. This pattern of bone imbalance contrasts with the increased bone resorption and subsequently increased formation observed in chronic ADT.

Table 1 Effect of androgen deprivation on general parameters and gonadal axis.

General parameters
BMI $\left(\mathrm{kg} / \mathrm{m}^{2}\right)$
Albumin $(\mathrm{g} / \mathrm{L})$
Hemoglobin $(\mathrm{g} / \mathrm{dL})$
eGFR (mL/min/1.73 $\left.\mathrm{m}^{2}\right)$
Cystatin C (mg/L)
ALP (IU/L)
AST (IU/L)
ALT (IU/L)
GGT (IU/L)
Gonadal axis
Total TS (nmol/L)
SHBG (nmol/L)
Free TS (nmol/L)
E2 (pmol/L)
LH (IU/L)
FSH (IU/L)

\begin{tabular}{c} 
Before ADT \\
\hline $24.6(17.9-31.7)$ \\
$46.0(41.2-50.3)$ \\
$15.1(13.1-16.3)$ \\
$101(63-131)$ \\
$0.87(0.77-1.27)$ \\
$68(54-109)$ \\
$21(12-28)$ \\
$21(13-53)$ \\
$15(10-71)$ \\
$17.8(8.3-25.2)$ \\
$37(18-71)$ \\
$0.32(0.18-0.43)$ \\
$61.3(31.6-129.2)$ \\
$5.4(2.3-26.5)$ \\
$3.6(1.9-53.5)$ \\
\hline
\end{tabular}

\begin{tabular}{c} 
After ADT \\
\hline $24.1(18.2-31.7)$ \\
$44.9(40.6-50.5)$ \\
$13.5(11.3-15.8)$ \\
$101(64-130)$ \\
$0.83(0.71-1.26)$ \\
$59(39-97)$ \\
$17(10-42)$ \\
$21(8.0-74)$ \\
$21(14-38)$ \\
$3.4(0.4-12.2)$ \\
$26(13-55)$ \\
$0.06(0.01-0.18)$ \\
$17.6(4.7-35.6)$ \\
$3.5(0.2-7.9)$ \\
$2.6(0.8-22.9)$
\end{tabular}

\begin{tabular}{r}
\multicolumn{1}{c}{ P-value } \\
\hline \\
0.6166 \\
0.0022 \\
0.0001 \\
$>0.9999$ \\
0.1697 \\
$<0.0001$ \\
0.2885 \\
0.7050 \\
0.0591 \\
$<0.0001$ \\
0.0009 \\
$<0.0001$ \\
$<0.0001$ \\
0.0040 \\
0.0025
\end{tabular}

Data are presented as median (range). Statistical significance was determined by a paired $t$-test if data were normally distributed, otherwise the Wilcoxon matched-pairs signed rank test was used (two-tailed $P<0.05$ ).

ALP, total alkaline phosphatase; ALT, alanine aminotransferase; AST, aspartate aminotransferase; E2, estradiol; eGFR, estimated glomerular filtration rate; GGT, gamma-glutamyl transferase; TS, testosterone. 

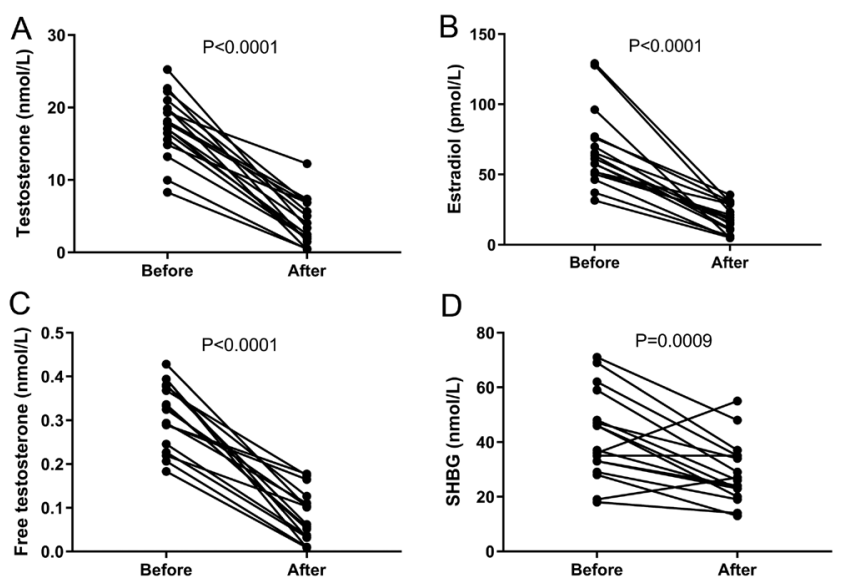

Figure 2

Change in sex steroids and SHBG after ADT by CPA. Each line represents one patient.

In this study, serum samples were taken from eugonadal male sex offenders referred to the endocrine clinic by their treating psychiatrist for initiation of chemical castration. Androgen deprivation with the AR antagonist CPA significantly decreased total $\mathrm{T}$ and E2. However, treatment with GnRH analogs might have suppressed T and E2 more prominently $(17,28)$.

Long-term sex steroid deprivation is characterized by increased bone formation markers at 6 months (15) and 1 year (16) following ADT in prostate cancer patients, as a result of coupling of formation to resorption. In addition, prostate cancer patients receiving $\mathrm{GnRH}$ analogs for a mean duration of 41 months also exhibited higher BAP levels than patients without ADT (3). In our study exploring
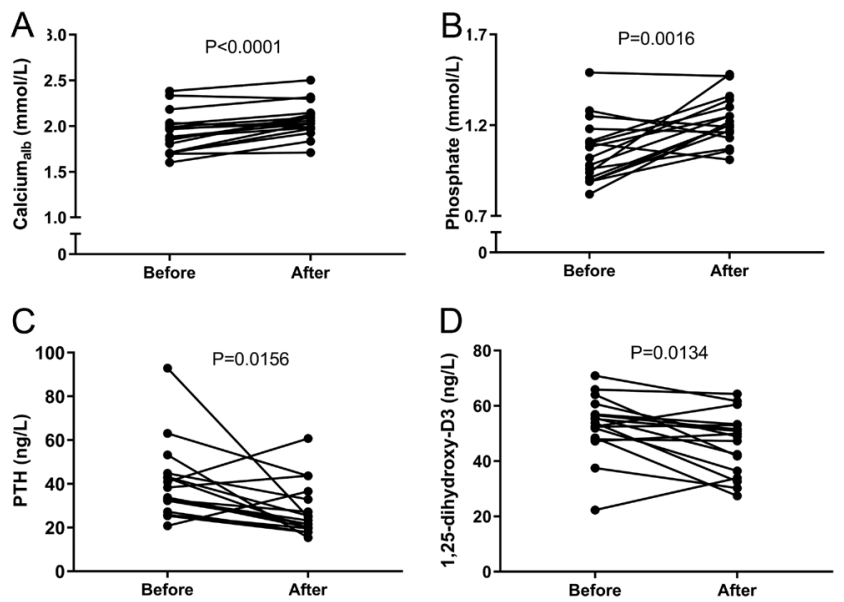

\section{Figure 3}

Change in mediators of calcium and phosphate homeostasis after ADT by CPA. Each line represents one patient. short-term effects of ADT on bone homeostasis, bone resorption did not appear to be increased, as indicated by unaltered TRAP and CTX concentrations. However, bone formation was reduced, as illustrated by the increase in sclerostin and decrease in BAP, osteocalcin and periostin. Sclerostin is produced by mature osteocytes, directly inhibiting bone formation and indirectly promoting bone resorption by enhancing osteoclast differentiation (29). Our data are in agreement with others, who described increased serum sclerostin after ADT in prostate cancer patients as well (30). In addition, young hypogonadal men have been shown to have increased serum sclerostin (31). Periostin, an extracellular matrix protein expressed by periosteal osteoblasts and osteocytes, promotes bone formation (32). It exerts its function by inhibiting sclerostin expression and stimulating the Wnt signaling pathway $(33,34)$. Accordingly, periostin-knockout mice exhibit both cortical and trabecular bone loss, leading to diminished bone strength (33). Serum periostin appears to be age-dependent, with higher concentrations in early adulthood than at older age (35). Moreover, serum concentrations have been associated with fracture risk in postmenopausal women (36). To our knowledge, we are the first to show that serum periostin may decrease early following ADT. This observation needs confirmation in a larger cohort.

Our results are in agreement with others who previously reported low bone formation in the early phase following ADT as well $(17,37)$. Interestingly, compared to studies in patients treated with GnRH analogs, the patients in our study were not severely estrogen-deficient, which could blunt the increase in bone resorption, known as being mainly estrogen-driven $(17,28)$. As such, CPA treatment might represent a model of mild androgen deprivation with more pronounced TS relative to estrogen deficiency. The coupling effect with bone formation, as generally seen with prolonged androgen and estrogen deficiency, may therefore not be present to the same extent in our study. As such, the observed acute impairment in bone formation could represent a dominant effect of TS. P1NP was not affected in our study. Discrepancies have been observed, with both unaltered (6) and increased (38) P1NP levels being reported following ADT. Timing could play a role, as others have described a decrease in serum P1NP after 4 weeks of ADT in healthy men, which normalized again by 12 weeks (37). Again, the extent of decrease in TS and E2 could play a role. Finkelstein et al. also showed that ADT with a GnRH analog in healthy men only affected P1NP when $\mathrm{T}$ was decreased below $3.5 \mathrm{nmol} / \mathrm{L}$ 
Table 2 Effect of androgen deprivation on calcium/phosphate homeostasis and bone turnover. Data are presented as median (range).

\begin{tabular}{l}
\hline \\
\hline Calcium/phosphate homeostasis \\
Calcium alb $(\mathrm{mmol} / \mathrm{L})$ \\
Phosphate $(\mathrm{mmol} / \mathrm{L})$ \\
PTH $(\mathrm{ng} / \mathrm{L})$ \\
Total $1.25 \mathrm{D}(\mathrm{ng} / \mathrm{L})$ \\
Total $25 \mathrm{D}(\mu \mathrm{g} / \mathrm{L})$ \\
Total $24.25 \mathrm{D}(\mathrm{ng} / \mathrm{mL})$ \\
25D:24.25D ratio \\
DBP $(\mu \mathrm{gg} / \mathrm{mL})$ \\
Free $1.25 \mathrm{D}(\mathrm{pg} / \mathrm{L})$ \\
Free $25 \mathrm{D}(\mathrm{ng} / \mathrm{L})$ \\
FGF23 $(\mathrm{pg} / \mathrm{mL})$ \\
Bone turnover \\
BAP $(\mu \mathrm{Hg} / \mathrm{L})$ \\
Osteocalcin $(\mathrm{ng} / \mathrm{mL})$ \\
P1 NP $(\mathrm{ng} / \mathrm{mL})$ \\
Periostin $(\mathrm{nmol} / \mathrm{L})$ \\
TRAP $(\mathrm{U} / \mathrm{L})$ \\
CTX $(\mathrm{ng} / \mathrm{L})$ \\
Sclerostin $(\mathrm{ng} / \mathrm{mL})$ \\
$\delta^{44 / 42 C a}(\% 0)$
\end{tabular}

\begin{tabular}{c}
\hline Before ADT \\
\hline $1.88(1.60-2.38)$ \\
$1.02(0.82-1.49)$ \\
$33.7(20.8-92.9)$ \\
$53.8(22.3-70.9)$ \\
$18.5(6.0-35.4)$ \\
$1.65(0.16-4.02)$ \\
$13.2(8.8-38.8)$ \\
$290(239-359)$ \\
$252(94-371)$ \\
$4.60(1.43-9.64)$ \\
$44.7(24.5-65.2)$ \\
$17.7(11.3-35.3)$ \\
$20.7(9.5-63.0)$ \\
$50.9(22.6-175)$ \\
$0.91(0.76-1.46)$ \\
$2.93(2.19-6.22)$ \\
$359(185-911)$ \\
$0.55(0.29-1.02)$ \\
$-0.77(-0.94$ to -0.58$)$ \\
\end{tabular}

\begin{tabular}{c} 
After ADT \\
\hline $2.03(1.71-2.50)$ \\
$1.20(1.01-1.48)$ \\
$23.2(15.4-60.7)$ \\
$48.9(27.4-64.3)$ \\
$23.0(11.7-43.7)$ \\
$2.04(0.74-4.53)$ \\
$10.4(7.0-15.8)$ \\
$314(269-393)$ \\
$205(118-283)$ \\
$5.90(2.66-10.3)$ \\
$45.4(25.8-78.5)$ \\
$12.7(6.2-20.7)$ \\
$18(7.0-37.4)$ \\
$55.4(19.2-109)$ \\
$0.87(0.561 .09)$ \\
$3.49(1.86-5.37)$ \\
$331(143-750)$ \\
$0.63(0.27-1.28)$ \\
$-0.88(-1.08$ to -0.56$)$ \\
\hline
\end{tabular}

$\begin{array}{r}\hline \text { P-value } \\ \hline \\ \hline 0.0001 \\ 0.0016 \\ 0.0156 \\ 0.0134 \\ 0.0496 \\ 0.0694 \\ 0.0785 \\ 0.0168 \\ 0.0041 \\ 0.1133 \\ 0.4737 \\ \\ <0.0001 \\ 0.0056 \\ 0.3529 \\ 0.0500 \\ 0.6441 \\ 0.8445 \\ <0.0001 \\ 0.0458 \\ \hline\end{array}$

Statistical significance was determined by a paired $t$-test if data were normally distributed, otherwise the Wilcoxon matched-pairs signed-rank test was used (two-tailed $P<0.05$ ).

1.25D, 1,25-dihydroxyvitamin $D_{3} ; 24.25 D, 24,25$-dihydroxyvitamin $D_{3} ; 25 D, 25$-hydroxyvitamin $D_{3} ; B A P$, bone alkaline phosphatase; $C T X, C$-terminal telopeptide of type I collagen; DBP, vitamin D-binding protein; FGF23, fibroblast growth factor 23; P1NP, N-terminal propeptide of type 1 collagen; TRAP, tartrate-resistant acid phosphatase $5 b ; \delta^{44 / 42} \mathrm{Ca}$, stable calcium isotope ratio.

(100 ng/dL) and E2 below $18 \mathrm{pmol} / \mathrm{L}(5 \mathrm{ng} / \mathrm{L})(28)$. In our study, approximately half of the men were above this threshold. Although P1NP and osteocalcin are both considered bone formation markers, they are expressed during different stages of osteoblast maturation (17). Therefore, as illustrated in this study, they may show a different response during the early stages of sex steroid deficiency.

Currently used bone resorption and formation markers may be influenced by formation and resorption, respectively, due to coupling. In contrast, the stable calcium isotope ratio reflects the net bone balance. We observed a shift toward lighter calcium isotopes, confirming the calcium flux from the skeleton. This marker has previously been shown to sensitively reflect a negative bone mineral balance in postmenopausal women with osteoporosis (26) as well as in response to skeletal unloading during long-term bed rest in males and females (27). Our data suggest that this novel calcium-based indicator of net bone mineral balance may also be used in male osteoporosis and represents a more sensitive marker for bone resorption in the early phase of ADT compared to CTX and TRAP. Early markers of bone resorption could be useful to identify patients who have a high risk of bone loss, such as prostate cancer patients and hypersexual men receiving ADT.

The increase in serum calcium and phosphate and concomitant decrease in PTH and 1,25-dihydroxyvitamin $\mathrm{D}_{3}$ are indicative for an acute net release of calcium and phosphate from the bone, consistent with the calcium isotope data. We failed to observe a compensatory increase of FGF23, probably due to a lack of power. Similar effects on calcium and phosphate homeostasis were reported following treatment with GnRH analogs in healthy adult men and prostate cancer patients after 12 weeks (39) and 6 months (7), respectively, with a secondary decrease in PTH $(7,39)$. Conversely, young adult and older hypogonadal men treated with androgen replacement showed decreased serum calcium (40). We observed a significant increase in DBP after androgen deprivation, thereby altering free vitamin $\mathrm{D}$ concentrations. In hypogonadal men on the other hand, $\mathrm{T}$ treatment has been shown to lower DBP after 4-12 weeks (41). In our study, free 1,25-dihydroxyvitamin $\mathrm{D}_{3}$ decreased to a greater extent than total 1,25-dihydroxyvitamin $\mathrm{D}_{3}$ and free 25-hydroxyvitamin $\mathrm{D}_{3}$ was unaffected. The 24,25-dihyroxyvitamin $\mathrm{D}_{3}$ levels and 25D:24,25D ratio were not changed either, indicative for a direct inhibitory 

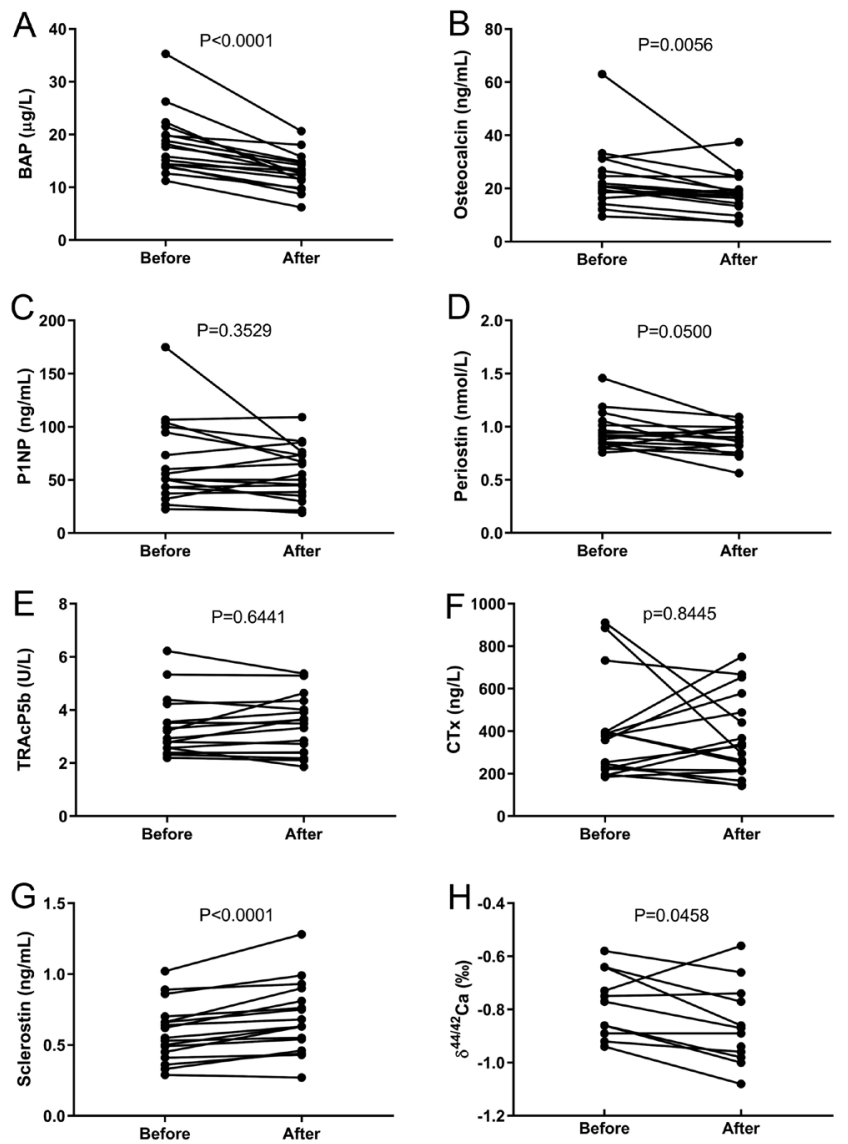

\section{Figure 4}

Change in bone turnover markers and stable calcium isotope ratio $\left(\delta^{44 / 42} \mathrm{Ca}\right)$ after ADT by CPA. Each line represents one patient.

effect of the decreased PTH on the $1 \alpha$-hydroxylase enzyme activity, thereby lowering the production of 1,25-dihydroxyvitamin $\mathrm{D}_{3}$.

Our study has several strengths. First, we prospectively investigated the effects of androgen deprivation in male sex offenders, which represents a younger patient group with less comorbidities compared to prostate cancer patients. Second, we explored the early effects of ADT on bone and calcium/phosphate homeostasis, including a wide range of BTMs and calcium-regulating hormones. Third, we assessed the serum calcium isotope ratio, a novel non-invasive method to sensitively detect early net calcium loss from the skeleton. Our study has several limitations as well, including the small number of participants, the absence of a control group, and the differences in timing of the follow-up visit. Replication of these findings is warranted in a larger and controlled clinical trial. Information on dietary calcium and phosphate intake and urine samples were not available, and blood samples were not systematically collected in a fasting state.

In summary, we explored the early effects of gonadal steroid withdrawal by CPA on the adult male skeleton and serum calcium and phosphate homeostasis. We observed that calcium is released from the skeleton, as detected by a novel stable calcium isotope technique and supported by a decrease of PTH and 1,25-dihydroxyvitamin $\mathrm{D}_{3}$. Concomitantly, serum sclerostin increased along with a reduction of bone formation markers, but without significant changes in bone resorption markers, suggesting that the negative calcium balance may be explained by an acute impairment of bone formation. This study helps in the understanding of the pathophysiology of acute hypogonadal bone loss in males.

In conclusion, the effects of short-term CPA treatment on skeletal dynamics in men are mediated largely, if not completely, by suppression of bone formation.

\section{Declaration of interest}

The authors declare that there is no conflict of interest that could be perceived as prejudicing the impartiality of this study.

\section{Funding}

This work was supported by the KU Leuven (grant GOA/15/017), the Research Foundation Flanders (FWO) (grant G0D2217N) and Klinische onderzoeks- en opleidingsraad (KOOR) University Hospitals Leuven (S54034).

\section{Author contribution statement}

D Vanderschueren and B Decallonne: Shared last author.

\section{Acknowledgments}

The authors would like to thank D Schollaert, E Van Herck, L Deboel and A Kolevica for excellent technical assistance, and S Achten for coordinating the study.

\section{References}

1 Almeida M, Laurent MR, Dubois V, Claessens F, O'Brien CA, Bouillon R, Vanderschueren D \& Manolagas SC. Estrogens and androgens in skeletal physiology and pathophysiology. Physiological Reviews 201797 135-187. (https://doi.org/10.1152/ physrev.00033.2015)

2 Wallander M, Axelsson KF, Lundh D \& Lorentzon M. Patients with prostate cancer and androgen deprivation therapy have increased risk of fractures-a study from the fractures and fall injuries in the elderly cohort (FRAILCO). Osteoporosis International 201930 115-125. (https://doi.org/10.1007/s00198-018-4722-3)

3 Stoch SA, Parker RA, Chen L, Bubley G, Ko YJ, Vincelette A \& Greenspan SL. Bone loss in men with prostate cancer treated with gonadotropin-releasing hormone agonists. Journal of Clinical 
Endocrinology and Metabolism 200186 2787-2791. (https://doi. org/10.1210/jcem.86.6.7558)

4 Wang A, Karunasinghe N, Plank L, Zhu S, Osborne S, Bishop K, Brown C, Schwass T, Masters J, Holmes M et al. Effect of androgen deprivation therapy on bone mineral density in a prostate cancer cohort in New Zealand: a pilot study. Clinical Medicine Insights: Oncology 201711 1179554917733449. (https://doi. org/10.1177/1179554917733449)

5 Greenspan SL, Coates P, Sereika SM, Nelson JB, Trump DL \& Resnick NM. Bone loss after initiation of androgen deprivation therapy in patients with prostate cancer. Journal of Clinical Endocrinology and Metabolism 200590 6410-6417. (https://doi. org/10.1210/jc.2005-0183)

6 Kimura T, Koike Y, Aikawa K, Kimura S, Mori K, Sasaki H, Miki K, Watanabe K, Saito M \& Egawa S. Short-term impact of androgen deprivation therapy on bone strength in castration-sensitive prostate cancer. International Journal of Urology 201926 980-984. (https://doi. org/10.1111/iju.14077)

7 Isahaya E, Hara N, Nishiyama T, Hoshii T, Takizawa I \& Takahashi K. Bone metabolic disorder in patients with prostate cancer receiving androgen deprivation therapy (ADT): impact of ADT on the growth hormone/insulin-like growth factor-1/parathyroid hormone axis. Prostate 201070 155-161. (https://doi.org/10.1002/pros.21047)

8 Dalla Via J, Daly RM, Owen PJ, Mundell NL, Rantalainen T \& Fraser SF. Bone mineral density, structure, distribution and strength in men with prostate cancer treated with androgen deprivation therapy. Bone 2019127 367-375. (https://doi.org/10.1016/j. bone.2019.06.005)

9 Poulsen MH, Frost M, Abrahamsen B, Gerke O, Walter S \& Lund L. Osteoporosis and prostate cancer; a 24-month prospective observational study during androgen deprivation therapy. Scandinavian Journal of Urology 201953 34-39. (https://doi.org/10.10 80/21681805.2019.1570328

10 Briken P \& Kafka MP. Pharmacological treatments for paraphilic patients and sexual offenders. Current Opinion in Psychiatry 200720 609-613. (https://doi.org/10.1097/YCO.0b013e3282f0eb0b)

11 Smid WJ, Kamphuis JH, Wever EC \& Verbruggen MCFM. Risk levels, treatment duration, and drop out in a clinically composed outpatient sex offender treatment group. Journal of Interpersonal Violence 2015 30 727-743. (https://doi.org/10.1177/0886260514536276)

12 Gooren LJ. Clinical review: ethical and medical considerations of androgen deprivation treatment of sex offenders. Journal of Clinical Endocrinology and Metabolism 201196 3628-3637. (https://doi. org/10.1210/jc.2011-1540)

13 Eastell R \& Szulc P. Use of bone turnover markers in postmenopausal osteoporosis. Lancet: Diabetes and Endocrinology 20175 908-923. (https://doi.org/10.1016/S2213-8587(17)30184-5)

14 Eastell R, Pigott T, Gossiel F, Naylor KE, Walsh JS \& Peel NFA. Bone turnover markers: are they clinically useful? European Journal of Endocrinology 2018178 R19-R31. (https://doi.org/10.1530/EJE-17-0585)

15 Ishizaki F, Hara N, Takizawa I, Nishiyama T, Isahaya E, Kawasaki T \& Takahashi K. Deficiency in androgens and upregulation of insulin-like growth factor-1 are involved in high bone turnover in men receiving androgen deprivation therapy for prostate cancer. Growth Hormone and IGF Research 201222 122-128. (https://doi. org/10.1016/j.ghir.2012.04.003)

16 Morote J, M'Hammed YID, Martinez E, Esquena S, Lorente JA \& Gelabert A. Increase of bone alkaline phosphatase after androgen deprivation therapy in patients with prostate cancer. Urology 200259 277-280. (https://doi.org/10.1016/s0090-4295(01)01518-7)

17 Falahati-Nini A, Riggs BL, Atkinson EJ, O'Fallon WM, Eastell R \& Khosla S. Relative contributions of testosterone and estrogen in regulating bone resorption and formation in normal elderly men. Journal of Clinical Investigation 2000106 1553-1560. (https://doi. org/10.1172/JCI10942)
18 Thibaut F, De La Barra FDL, Gordon H, Cosyns P \& Bradford JMW. The World Federation of Societies of Biological Psychiatry (WFSBP) guidelines for the biological treatment of paraphilias. World Journal of Biological Psychiatry 201011 604-655. (https://doi. org/10.3109/15622971003671628)

19 Assumpção AA, Garcia FD, Garcia HD, Bradford JMW \& Thibaut F. Pharmacologic treatment of paraphilias. Psychiatric Clinics of North America 201437 173-181. (https://doi.org/10.1016/j.psc.2014.03.002)

20 Antonio L, Pauwels S, Laurent MR, Vanschoubroeck D, Jans I, Billen J, Claessens F, Decallonne B, De Neubourg D, Vermeersch P et al. Free testosterone reflects metabolic as well as ovarian disturbances in subfertile oligomenorrheic women. International Journal of Endocrinology 20182018 7956951. (https://doi. org/10.1155/2018/7956951)

21 Pauwels S, Antonio L, Jans I, Lintermans A, Neven P, Claessens F, Decallonne B, Billen J, Vanderschueren D \& Vermeersch P. Sensitive routine liquid chromatography-tandem mass spectrometry method for serum estradiol and estrone without derivatization. Analytical and Bioanalytical Chemistry 2013405 8569-8577. (https://doi. org/10.1007/s00216-013-7259-5)

22 Vermeulen A, Verdonck L \& Kaufman JM. A critical evaluation of simple methods for the estimation of free testosterone in serum. Journal of Clinical Endocrinology and Metabolism 199984 3666-3672. (https://doi.org/10.1210/jcem.84.10.6079)

23 Cools M, Goemaere S, Baetens D, Raes A, Desloovere A, Kaufman JM, De Schepper J, Jans I, Vanderschueren D, Billen J et al. Calcium and bone homeostasis in heterozygous carriers of CYP24A1 mutations: a cross-sectional study. Bone 201581 89-96. (https://doi.org/10.1016/j. bone.2015.06.018)

24 Casetta B, Jans I, Billen J, Vanderschueren D \& Bouillon R. Development of a method for the quantification of $1 \alpha, 25(\mathrm{OH})$ 2 - vitamin D 3 in serum by liquid chromatography tandem mass spectrometry without derivatization. European Journal of Mass Spectrometry 201016 81-89. (https://doi.org/10.1255/ejms.1024)

25 Bouillon R, Vandoren G, Van Baelen H \& De Moor P. Immunochemical measurement of the vitamin D-binding protein in rat serum. Endocrinology 1978102 1710-1715. (https://doi. org/10.1210/endo-102-6-1710)

26 Eisenhauer A, Müller M, Heuser A, Kolevica A, Glüer CC, Both M, Laue C, Hehn UV, Kloth S, Shroff R et al. Calcium isotope ratios in blood and urine: a new biomarker for the diagnosis of osteoporosis. Bone Reports 201910 100200. (https://doi.org/10.1016/j. bonr.2019.100200)

27 Channon MB, Gordon GW, Morgan JLL, Skulan JL, Smith SM \& Anbar AD. Using natural, stable calcium isotopes of human blood to detect and monitor changes in bone mineral balance. Bone 201577 69-74. (https://doi.org/10.1016/j.bone.2015.04.023)

28 Finkelstein JS, Lee H, Leder BZ, Burnett-Bowie SA, Goldstein DW, Hahn CW, Hirsch SC, Linker A, Perros N, Servais AB et al. Gonadal steroid-dependent effects on bone turnover and bone mineral density in men. Journal of Clinical Investigation 2016126 1114-1125. (https://doi.org/10.1172/JCI84137)

29 Holdsworth G, Roberts SJ \& Ke HZ. Novel actions of sclerostin on bone. Journal of Molecular Endocrinology 201962 R167-R185. (https:// doi.org/10.1530/JME-18-0176)

30 Garcia-Fontana B, Morales-Santana S, Varsavsky M, Garcia-Martin A, Garcia-Salcedo JA, Reyes-Garcia R \& Munoz-Torres M. Sclerostin serum levels in prostate cancer patients and their relationship with sex steroids. Osteoporosis International 201425 645-651. (https://doi. org/10.1007/s00198-013-2462-y)

31 Di Nisio A, De Toni L, Speltra E, Rocca MS, Taglialavoro G, Ferlin A $\&$ Foresta C. Regulation of sclerostin production in human male osteocytes by androgens: experimental and clinical evidence. Endocrinology 2015156 4534-4544. (https://doi.org/10.1210/ en.2015-1244) 
32 Bonnet N, Garnero P \& Ferrari S. Periostin action in bone. Molecular and Cellular Endocrinology 2016432 75-82. (https://doi.org/10.1016/j. mce.2015.12.014)

33 Bonnet N, Standley KN, Bianchi EN, Stadelmann V, Foti M, Conway SJ \& Ferrari SL. The matricellular protein periostin is required for sost inhibition and the anabolic response to mechanical loading and physical activity. Journal of Biological Chemistry 2009 284 35939-35950. (https://doi.org/10.1074/jbc.M109.060335)

34 Bonnet N, Conway SJ \& Ferrari SL. Regulation of beta catenin signaling and parathyroid hormone anabolic effects in bone by the matricellular protein periostin. PNAS 2012109 15048-15053. (https://doi.org/10.1073/pnas.1203085109)

35 Walsh JS, Gossiel F, Scott JR, Paggiosi MA \& Eastell R. Effect of age and gender on serum periostin: relationship to cortical measures, bone turnover and hormones. Bone 201799 8-13. (https://doi. org/10.1016/j.bone.2017.03.041)

36 Rousseau JC, Sornay-Rendu E, Bertholon C, Chapurlat R \& Garnero P. Serum periostin is associated with fracture risk in postmenopausal women: a 7-year prospective analysis of the OFELY study. Journal of Clinical Endocrinology and Metabolism 201499 2533-2539. (https:// doi.org/10.1210/jc.2013-3893)

37 Leder BZ, LeBlanc KM, Schoenfeld DA, Eastell R \& Finkelstein JS. Differential effects of androgens and estrogens on bone turnover in normal men. Journal of Clinical Endocrinology and Metabolism 200388 204-210. (https://doi.org/10.1210/jc.2002-021036)

38 Hamilton EJ, Ghasem-Zadeh A, Gianatti E, Lim-Joon D, Bolton D, Zebaze R, Seeman E, Zajac JD \& Grossmann M. Structural decay of bone microarchitecture in men with prostate cancer treated with androgen deprivation therapy. Journal of Clinical Endocrinology and Metabolism 201095 E456-E463. (https://doi.org/10.1210/jc.20100902)

39 Burnett-Bowie SM, Mendoza N \& Leder BZ. Effects of gonadal steroid withdrawal on serum phosphate and FGF-23 levels in men. Bone 200740 913-918. (https://doi.org/10.1016/j. bone.2006.10.016)

40 Katznelson L, Finkelstein JS, Schoenfeld DA, Rosenthal DI, Anderson EJ \& Klibanski A. Increase in bone density and lean body mass during testosterone administration in men with acquired hypogonadism. Journal of Clinical Endocrinology and Metabolism 199681 4358-4365. (https://doi.org/10.1210/ jcem.81.12.8954042)

41 Hagenfeldt Y, Linde K, Sjoberg HE, Zumkeller W \& Arver S. Testosterone increases serum 1,25-dihydroxyvitamin D and insulin-like growth factor-I in hypogonadal men. International Journal of Andrology 199215 93-102. (https://doi. org/10.1111/j.1365-2605.1992.tb01118.x)

Received 10 April 2020

Revised version received 20 May 2020

Accepted 26 May 2020 